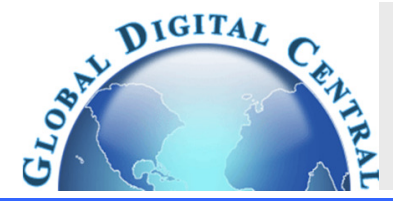

Frontiers in Heat and Mass Transfer

Available at www.ThermalFluidsCentral.org

\title{
EFFECTS OF THERMAL AND SOLUTAL STRATIFICATION ON MIXED CONVECTION FLOW ALONG A VERTICAL PLATE SATURATED WITH COUPLE STRESS FLUID
}

\author{
K. Kaladhar ${ }^{\mathrm{a}}$, D. Srinivasacharya ${ }^{\mathrm{b}}$ \\ ${ }^{a}$ Department of Mathematics, National Institute of Technology Puducherry, Karaikal-609605, India \\ ${ }^{b}$ Department of Mathematics, National Institute of Technology, Warangal-506004, India
}

\begin{abstract}
The effect of heat and mass stratification on mixed convection along a vertical plate embedded in a couple stress fluid has been presented. The nonlinear system of equations with appropriate boundary conditions is primarily reduced to non-dimensional form by pseudo-similarity transformations. Keller-box implicit finite difference scheme is employed to solve the resultant system of dimensionless equations. The validation of this scheme is shown through the comparison between the present and available literature under special case of the present problem. The couple stress parameter, mixed convection parameter and the double stratification parameter effects on the rates of heat and mass transfer for diverse values of the emerging flow parameters are illustrated in tabular form. The present results show that the couple stress parameter, mixed convection parameter, thermal and the solutal stratification parameters influences the flow significantly.
\end{abstract}

Keywords: Mixed convection, couple stress fluid, double stratification.

\section{INTRODUCTION}

A flow with free and forced convection is of considerable interest due to its wide range in manufacturing and practical applications that consist of, metallurgical processes, nuclear reactors, heat exchangers, geothermal systems, geothermal systems, crystal growth, nuclear waste materials, and many. In literature, convection in heat and mass transfer flow along a non-isothermal vertical plane for Newtonian fluids with boundary layer estimates have been studied by many authors. The theoretical results for heat and solutal transfer from a vertical flat plate have been presented by Somers (1956). Szewczyk (1964) analyzed the effects of mixed convection laminar flow. Mixed convection flow along a flat plate with local similarity method was studied by Lloyd and Sparrow (1970). It has been produced the solutions through pure to mixed convection. Detailed explanation of many works can be found in Bejan (1994). Most recently, The effect on homogeneous chemical reaction on mixed convection in a porous medium saturated with polar fluid have been studied numerically by Patil and Chamkha (2013).

Analysis of non-Newtonian fluids with heat and mass transfer is momentous in practical situations. For instance, slurries, foodstuffs, polymeric liquids, thermal design of industrial equipment dealing with molten plastics, etc. The examples of such fluids are blood at low shear rate, lubricants containing small amount of polymer additives, electrorheological fluids, paints, fiber solutions and synthetic fluids, etc. The nonlinear relationship between the rate of strain and stress can be found in non-Newtonian fluid models. Stokes (1966) introduced this model, in which body couples, non-symmetric tensors and couple stresses exist. The classical viscous theories fails to describe the size dependant effect but which can be found with the effect of couple stresses. The free and mixed convection flow of couple stress fluid in a vertical channel have been presented by Srinivasacharya and Kaladhar (2012a,b). The magnetohydrodynamics, viscous dissipation and heat mass transfer effects on horizontal wavy channel in a porous channel saturated with couple stress fluid have been discussed by Muthuraj et al. (2013). The

* Corresponding author. Email: kkr.nitpy@gmail.com couple stress fluid with melting heat transfer under stagnation point flow was offered by Hayat et al. (2013). Srinivasacharya and Kaladhar (2013) described the analytical solution for mixed convection flow of couple stress fluid with MHD, Hall and ion-slip effects between circular rotating parallel disks. Makinde and Eegunjobi (2013) studied the nature of couple stress fluid in a vertical channel with entropy generation and porous medium. Najeeb et al. (2013) reported the flow of couple stress fluid in an contracting and expanding porous channel with an approximate solutions. Most recently, the size-dependent and consisting creeping flow of couple stress fluid was presented by Hadjesfandiari et al. (2013). The presence of different fluids or variations in temperature/ concentration leads to the stratification of a fluid. Although there is minute literature and in view of applications, Prandtl (1952), Jaluria and Himasekhar (1983), Murthy et al. (2004), Srinivasacharya et al. (2011) and few more are presented the stratification effects in different cases.

It is the objective of the present work to consider the mixed convection flow of couple stress fluid along a vertical plate with solutal and thermal stratification effects. The medium is linearly stratified and the wall concentration and wall temperature are constants. The present boundary conditions are close realistic to the practical interest, like the heat mass transfer characteristics around a cooling magmatic intrusion or around a hot radioactive subsurface storage site where the theory of convection is involved. The governing nonlinear system of equations are solved by using the Keller box method (Cebeci and Bradshaw (1984)). The effects of the couple stress parameter, mixed convection, and the stratification parameters are examined and are presented graphically.

\section{ANALYSIS}

The geometry of the problem and the coordinate system are shown in Fig. 1, in which $x$-axis and $y$-axis are along and normal to the vertical plate respectively. Steady and incompressible couple stress fluid along a plat plate is considered. Stratification and mixed convection are also taken into consideration. Density change in the fluid is neglected 
everywhere except in the buoyancy, and all the other physical properties of the fluid are assumed constant. The uniform temperature and concentrations of the plate is $T_{w}$ and $C_{w}$ respectively. The free stream velocity, temperature and concentration parallel to the vertical plate are maintained at $u_{\infty}, T_{\infty, 0}$ and $C_{\infty, 0}$ respectively. At any arbitrary reference point in the medium (inside the boundary layer), the values of $T_{\infty, 0}$ and $C_{\infty, 0}$ are smaller than $T_{w}$ and $C_{w}$ respectively. $T_{\infty}(x)=T_{\infty, 0}+A x$ and $C_{\infty}(x)=C_{\infty, 0}+B x$ are the vertical linear temperature and concentration stratification parameters in an ambient medium. Where $A$ and $B$ are constants, these are varied to alter the intensity of stratification in the medium.

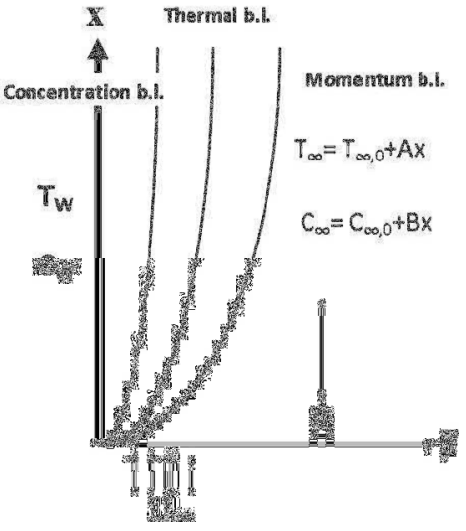

Fig 1 Physical model and coordinate system

The governing equations for the couple stress fluid, boundary layer approximations and Boussinesq approximations are given by

$$
\begin{aligned}
& \frac{\partial u}{\partial x}+\frac{\partial v}{\partial y}=0 \\
& \rho\left(u \frac{\partial u}{\partial x}+v \frac{\partial u}{\partial y}\right)=\mu \frac{\partial^{2} u}{\partial y^{2}}+\rho g\left(\beta_{T}\left(T-T_{\infty}\right)+\beta_{C}\left(C-C_{\infty}\right)\right)-\eta_{1} \frac{\partial^{4} u}{\partial y^{4}} \\
& u \frac{\partial T}{\partial x}+v \frac{\partial T}{\partial y}=\alpha \frac{\partial^{2} T}{\partial y^{2}} \\
& u \frac{\partial C}{\partial x}+v \frac{\partial C}{\partial y}=D \frac{\partial^{2} C}{\partial y^{2}}
\end{aligned}
$$

where the velocities in $x$ and $y$ directions are $u$ and $v$ respectively, $T$ is the temperature, $C$ is the concentration, $g$ is the acceleration due to gravity, $\rho$ is the density, $\mu$ is the dynamic coefficient of viscosity, $\beta_{T}$ is the coefficient of thermal expansion, $\beta_{C}$ is the coefficient of solutal expansions, $\alpha$ is the thermal diffusivity and $D$ is the solutal diffusivity of the medium and $\eta_{1}$ is the couple stress fluid parameter. The subscript $\infty$ indicates the condition at the outer edge of the boundary layer.

$$
\begin{aligned}
& \text { The physical boundary conditions for this problem are } \\
& u=0, v=0, v_{x}-u_{y}=0, T=T_{w}, C=C_{w} \text { at } y=0 \\
& u=0, v_{x}-u_{y}=0, T=T_{\infty}(x), C=C_{\infty}(x) \text { as } y \rightarrow \infty
\end{aligned}
$$

where the boundary condition $v_{x}-u_{y}=0$ imply that the couple stress fluid is irrotational at the boundaries (Type $A$ condition for Couple stress fluid; Stokes (1966)) and $k$ is the thermal conductivity of the fluid.

The stream function $\psi$ is introduced in view of continuity equation (1), which is

$$
u=\frac{\partial \psi}{\partial y}, \quad v=-\frac{\partial \psi}{\partial x}
$$

Substituting Eq. (6) in Eqs. (2)-(4) and then using the following transformations

$$
\begin{aligned}
& \xi=\frac{\mathrm{x}}{\mathrm{L}}, \eta=\left(\frac{\mathrm{Re}}{\xi}\right)^{\frac{1}{2}} \frac{y}{L}, f(\xi, \eta)=\left(\frac{\mathrm{Re}}{\xi}\right)^{\frac{1}{2}} \frac{\psi}{L u_{\infty}} \\
& \theta(\xi, \eta)=\frac{T-T_{\infty, 0}}{T_{w}-T_{\infty, 0}}-\frac{A x}{T_{w}-T_{\infty, 0}} \\
& \phi(\xi, \eta)=\frac{C-C_{\infty, 0}}{C_{w}-C_{\infty, 0}}-\frac{B x}{C_{w}-C_{\infty, 0}}
\end{aligned}
$$

The final non-linear system of differential equations is

$$
\begin{aligned}
& \operatorname{Re} S^{2} f^{v}-\xi f^{\prime \prime \prime}-\frac{1}{2} \xi f f^{\prime \prime}-\xi^{2} \operatorname{Ri}(\theta+\mathrm{N} \varphi)=\xi^{2}\left(f^{\prime \prime} \frac{\partial f}{\partial \xi}-f^{\prime} \frac{\partial f^{\prime}}{\partial \xi}\right) \\
& \frac{1}{\operatorname{Pr}} \theta^{\prime \prime}+\frac{1}{2} f \theta^{\prime}-\varepsilon_{1} \xi f^{\prime}=\xi\left(f^{\prime} \frac{\partial \theta}{\partial \xi}-\theta^{\prime} \frac{\partial f}{\partial \xi}\right) \\
& \frac{1}{S c} \phi^{\prime \prime}+\frac{1}{2} f \phi^{\prime}-\varepsilon_{2} \xi f^{\prime}=\xi\left(f^{\prime} \frac{\partial \phi}{\partial \xi}-\phi^{\prime} \frac{\partial f}{\partial \xi}\right)
\end{aligned}
$$

where the primes indicate partial differentiation with respect to $\eta$ alone, $\operatorname{Re}=\frac{u_{\infty} L}{v}$ is the Reynolds number, $G r=\frac{g \beta_{T}\left(T_{w}-T_{\infty, 0}\right) L^{3}}{v^{2}}$ is the thermal Grashof number, $\operatorname{Pr}=\frac{v}{\alpha} \quad$ is the Prandtl number, $S c=\frac{v}{D}$ is the Schmidt number, $\eta_{l}$ is the couple stress fluid parameter and $N=\frac{\beta_{C}\left(C_{w}-C_{\infty, 0}\right)}{\beta_{T}\left(T_{w}-T_{\infty, 0}\right)}$ is the buoyancy ratio. It should be noted that $N>0$ indicates aiding buoyancy where both the thermal and solutal buoyancies are in the same direction and $N<0$ indicates opposing buoyancy where the solutal buoyancy is in the opposite direction to the thermal buoyancy. When $N=0$, the flow is driven by thermal buoyancy alone. $R i=\frac{G r}{\mathrm{Re}^{2}}$ is the mixed convection parameter, which represents the ratio of buoyancy forces to the inertia forces and is used to delineate the free, forced and mixed convection regimes. $R i<<1$ corresponds to pure forced convection, whereas $R i \gg 1$ corresponds to pure free convection. $\varepsilon_{1}=\frac{A L}{T_{w}-T_{\infty, 0}}$ and $\varepsilon_{2}=\frac{B L}{C_{w}-C_{\infty, 0}}$ are the thermal and solutal stratification parameters and are constants. $S=\frac{1}{L} \sqrt{\frac{\eta_{1}}{\mu}}$ is the couple stress parameter, the effects of couple-stress are significant for large values of $S(=l / L)$, where $l=\sqrt{\frac{\eta_{1}}{\mu}}$ is the material constant. If

Boundary conditions (5) in terms of $f, \theta$ and $\phi$ become

$$
\begin{array}{r}
\eta=0: f^{\prime}(\xi, 0)=0, f(\xi, 0)=f^{\prime \prime}(\xi, 0)=0, \theta(\xi, 0)=1-\varepsilon_{1} \xi, \\
\varphi(\xi, 0)=1-\varepsilon_{2} \xi
\end{array}
$$

(11a)

$$
\eta \rightarrow \infty: f^{\prime}(\xi, \infty)=1, \quad f^{\prime \prime}(\xi, \infty)=0, \theta(\xi, \infty)=0, \phi(\xi, \infty)=0
$$


by

The heat and mass transfers from the plate are given respectively

$$
q_{w}=-k\left[\frac{\partial T}{\partial y}\right]_{y=0}, q_{m}=-D\left[\frac{\partial C}{\partial y}\right]_{y=0}
$$

The local Nusselt number $N u_{x}=\frac{q_{w} x}{k\left(T_{w}-T_{\infty, 0}\right)}$ and local Sherwood number $S h_{x}=\frac{q_{m} x}{D\left(C_{w}-C_{\infty, 0}\right)}$ are given by

$$
N u_{x}=-\operatorname{Re}_{x}^{1 / 2} \theta^{\prime}(\xi, 0), S h_{x}=-\operatorname{Re}_{x}^{1 / 2} \varphi^{\prime}(\xi, 0)
$$

where $\operatorname{Re}_{x}=\frac{u_{\infty} x}{v}$ is the local Reynolds number.

\section{RESULTS AND DISCUSSIONS}

The energy equation (9) and concentration (10) are coupled with the flow equation (8). Since the system of differential equations is nonlinear and non-homogenous, the closed form solutions are not obtained and hence the system is solved using the implicit Keller-box method (Cebeci and Bradshaw (1984)). This method has been proven to be adequate and give accurate results for boundary layer equations. In boundary conditions, the value of $\eta$ at $\infty$ is replaced by a sufficiently large value of $\eta$ where the temperature, concentration profiles approach zero and velocity approaches to one. For that the value is considered as $\eta=8$ with a grid size of $\eta$ of 0.01 . The dimensionless velocity, temperature and concentration function are evaluated and are presented through plots, which are shown in Figs. 2-13. The effects of emerging parameters of the flow on dimensionless velocity, temperature and concentration are discussed. In throughout the computations the constant values are assumed as $\operatorname{Re}=100, \operatorname{Pr}=0.71, S c=0.22$ and $\xi$ $=0.1$.

By taking $S=0, \varepsilon_{1}=0, \varepsilon_{2}=0$, and $N=0$, the present system of equations reduces to Newtonian fluid with mixed convection flow of vertical plate of Lloyd and Sparrow (1970).

Table 1: Comparison of $N u_{x} R e_{x}^{-1 / 2}$ for mixed convection between a vertical flat plate and Newtonian fluids (Lloyd and Sparrow (1970))

\begin{tabular}{l|l|l|l|l|l|l}
\hline \multicolumn{1}{c}{$\operatorname{Pr}=0.72$} & \multicolumn{2}{c}{$\operatorname{Pr}=10$} & \multicolumn{3}{c}{$\operatorname{Pr}=100$} \\
\hline $\mathrm{Ri}$ & $\begin{array}{l}\text { Lloyd } \\
\text { and } \\
\text { Sparrow }\end{array}$ & Present & $\begin{array}{l}\text { Lloyd } \\
\text { and } \\
\text { Sparrow }\end{array}$ & Present & $\begin{array}{l}\text { Lloyd } \\
\text { and } \\
\text { Sparrow }\end{array}$ & Present \\
\hline 0.00 & 0.2956 & 0.2956 & 0.7281 & 0.7281 & 1.5720 & 1.5743 \\
0.01 & 0.2979 & 0.2979 & 0.7313 & 0.7313 & 1.5750 & 1.5766 \\
0.04 & 0.3044 & 0.3044 & 0.7404 & 0.7410 & 1.5850 & 1.5891 \\
0.10 & 0.3158 & 0.3158 & 0.7574 & 0.7581 & 1.6050 & 1.6065 \\
0.40 & 0.3561 & 0.3561 & 0.8259 & 0.8272 & 1.6910 & 1.6962 \\
1.00 & 0.4058 & 0.4058 & 0.9212 & 0.9212 & 1.8260 & 1.8293 \\
2.00 & 0.4584 & 0.4584 & 1.0290 & 1.0302 & 1.9940 & 1.9985 \\
4.00 & 0.5258 & 0.5258 & 1.1730 & 1.1738 & 2.2320 & 2.2385 \\
\hline
\end{tabular}

Thus, using the principle of local similarity and in absence of couple stress parameter $S$, buoyancy ratio, stratification parameters $\varepsilon_{1}$ and $\varepsilon_{2}$ with $\xi=1$, the comparison has been made by the special case of Lloyd and Sparrow (1970) with the outcome of the governing equations (2)-(4) and it is noticed that they are in good agreement, as shown in Table 1. Therefore, the present code has been used with great confidence to this study.

The dimensionless velocity profile for different values of mixed convection parameter at fixed $S, \varepsilon_{1}$ and $\varepsilon_{2}$ is shown in Fig. 2. It can be observed that the dimensionless velocity rises as the mixed convection parameter increases. Compared with the pure forced convection case ( $R i=0.0$, i.e. the limiting case), higher values of $R i$ implies the higher velocity. This is because of increase in mixed convection parameter leads to the higher buoyancy effects in mixed convection and on temperature profile is examined in Fig. 3. It can be seen from this figure that as $R i$ increases, the dimensionless temperature decreases. By comparing with pure forced convection the temperature is less in case of mixed convection. The temperature reduces when the convection cooling effect increases, this happens when $R i$ (buoyancy effects) increases. Figure 4 depicts that the concentration profile at different values of $R i$. It is noticed that as $R i$ increases, the concentration decreases.

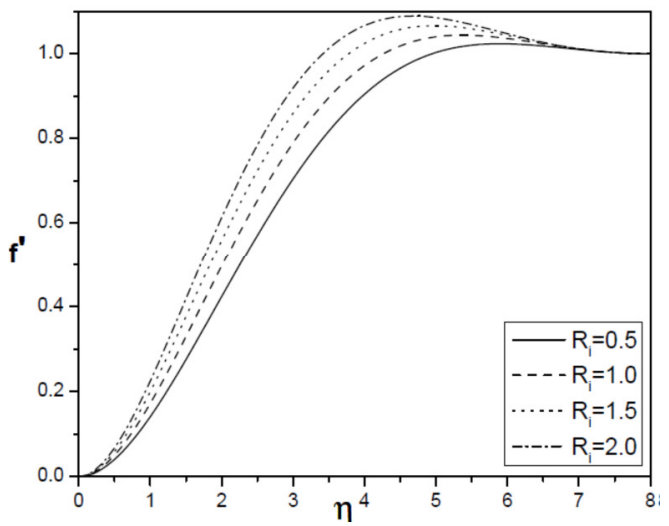

Fig. 2 Effect of mixed convection parameter $R i$ on velocity when $S=0.5, \varepsilon_{1}=0.1, \varepsilon_{2}=0.1$

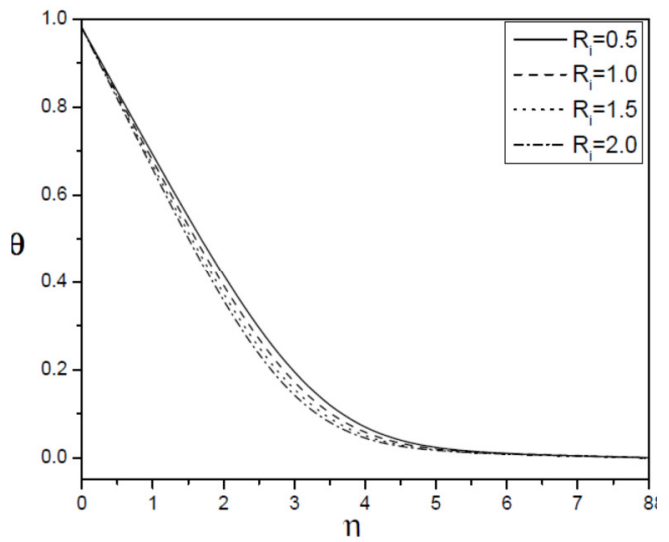

Fig. 3 Effect of mixed convection parameter Ri on Temperature when $S=0.5, \varepsilon_{1}=0.1, \varepsilon_{2}=0.1$

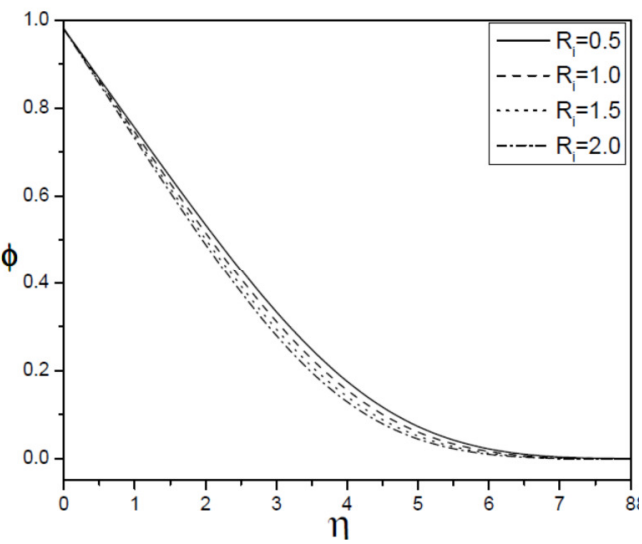

Fig. 4 Effect of mixed convection parameter $R i$ on Concentration when $S=0.5, \varepsilon_{1}=0.1, \varepsilon_{2}=0.1$ 


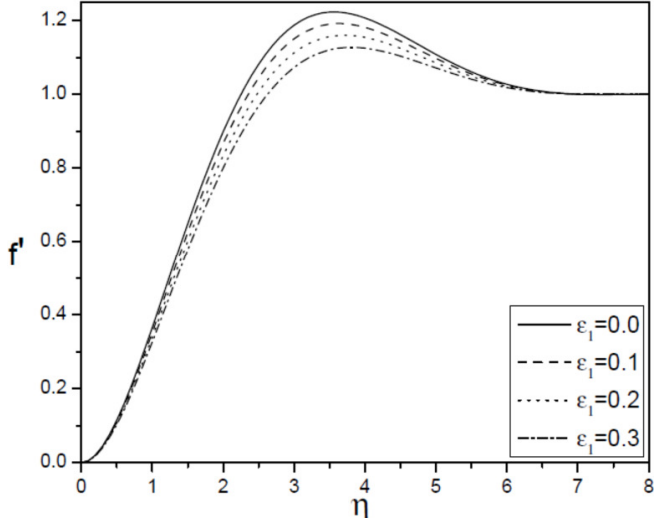

Fig. 5 Effect of thermal stratification parameter $\varepsilon_{1}$ on velocity when $S=0.5, R i=2.0, \varepsilon_{2}=0.1$

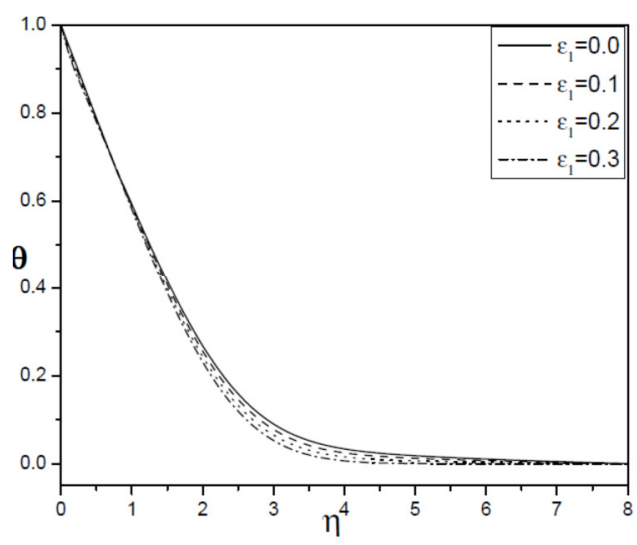

Fig. 6 Effect of thermal stratification parameter $\varepsilon_{1}$ on velocity when $S=0.5, R i=2.0, \varepsilon_{2}=0.1$

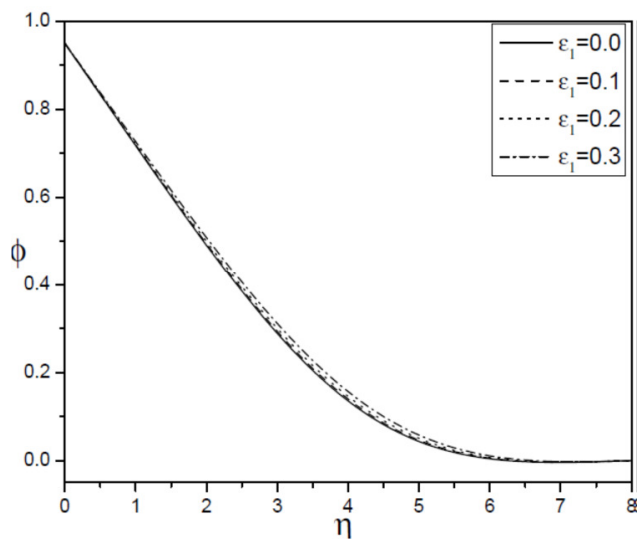

Fig. 7 Effect of thermal stratification parameter $\varepsilon_{1}$ on velocity when $S=0.5, R i=2.0, \varepsilon_{2}=0.1$

The effect of stratification parameter $\varepsilon_{1}$ on non-dimensional velocity is depicted in Fig. 5. It can be seen that as the temperature stratification increases, the velocity decreases. As there is any decrease in thermal stratification, the effective convective potential between the heated plate and the ambient fluid in the medium reduces. Therefore under the boundary layer the velocity reduces with the thermal stratification. Figure 6 shows that the effect of $\varepsilon_{1}$ on temperature profile for fixed values of $S, R i$ and $\varepsilon_{2}$. It can be found that increase in $\varepsilon_{1}$ leads the decrease in dimensionless temperature. The effective temperature difference between the plate and the ambient fluid will decrease under the influence of thermal stratification effect. Hence the temperature reduces as the thermal boundary layer thickened. At fixed values of $S=$
$0.5, R i=2$ and $\varepsilon_{2}=0.1$, the thermal stratification parameter effect on concentration profile is shown in Fig. 7. It is observed that the dimensionless concentration increases with an increase in $\varepsilon_{1}$.

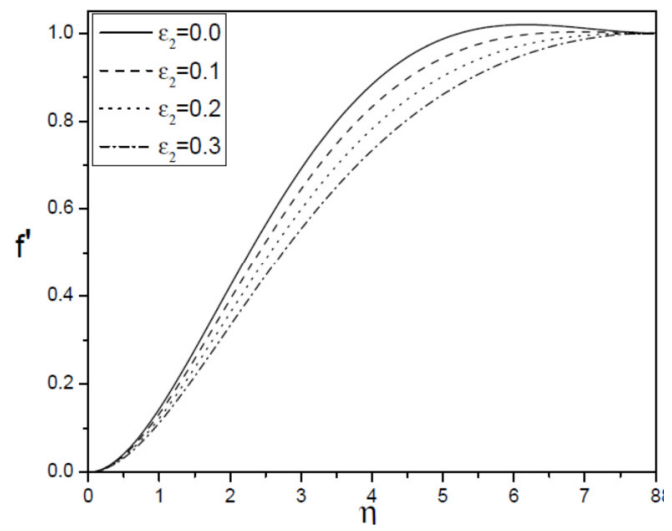

Fig. 8 Effect of solutal stratification parameter $\varepsilon_{2}$ on velocity when $S=0.5, R i=2.0, \varepsilon_{1}=0.05$

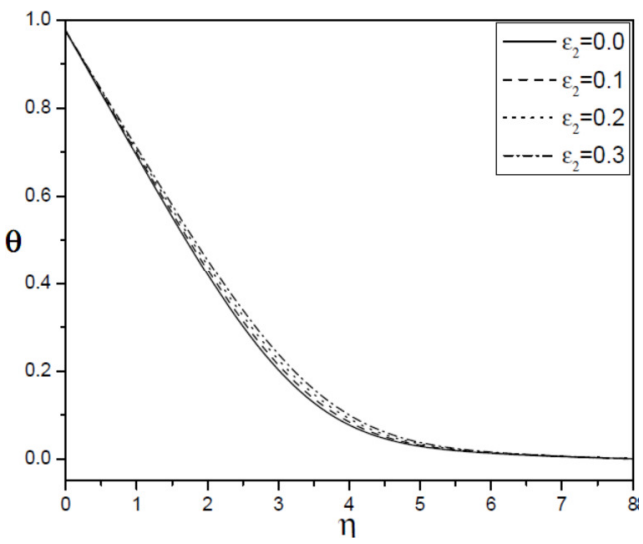

Fig. 9 Effect of solutal stratification parameter $\varepsilon_{2}$ on velocity when $S=0.5, R i=2.0, \varepsilon_{1}=0.05$

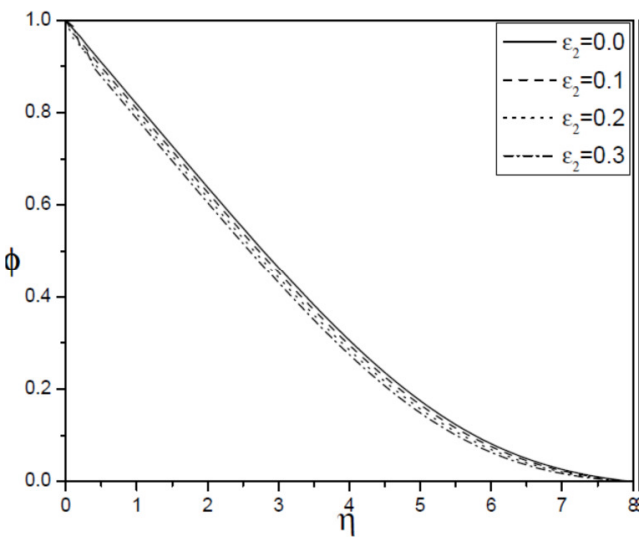

Fig. 10 Effect of solutal stratification parameter $\varepsilon_{2}$ on velocity when $S=0.5, R i=2.0, \varepsilon_{1}=0.05$

Figure 8 analyzes the effect of $\varepsilon_{2}$ on the velocity profile by taking $S=0.5, R i=2$ and $\varepsilon_{1}=0.05$. We see that the dimensionless velocity decreases as an increase in $\varepsilon_{2}$. Figure 9 represents the $\varepsilon_{2}$ effect on dimensionless temperature profile at $S=0.5, R i=2$ and $\varepsilon_{1}=0.05$. As the solutal stratification increases, the dimensionless temperature profile increases. The effect of $\varepsilon_{2}$ on concentration profile can be seen in Fig. 
10. It can be seen from the figure that as an increase in $\varepsilon_{2}$ leads to decrease in concentration of the fluid.

In Figs. 11-13, the profiles of the dimensionless velocity, temperature and concentration is shown with the influence of couple stress parameter $S$ for fixed values of $R i=2.0, \varepsilon_{1}=0.1$ and $\varepsilon_{2}=0.1$. It can be seen that the maximum velocity decreases in amplitude and the location of the maximum velocity moves far away from the wall as an increase in $S$. Since in couple stress fluid the rotational field of the velocity will be amplified. Also, as there is an increase in $S$, the temperature profile increases. It is also observed that the concentration profile increases as an increase in $S$.

The dimensionless heat and mass transfer rates are presented in Table 2 at different values of $R i, \varepsilon_{1}$ and $\varepsilon_{2}$ for fixed value of $S=0.5$. It is observed that increase in $\varepsilon_{1}$ leads to increase in heat transfer coefficient and decrease in mass transfer coefficient. But in the case of increase in $\varepsilon_{2}$, the reverse trend is found. Also the effect of $R i$ on Nusselt and Sherwood is shown in the same Table 2 with fixed values of $\varepsilon_{1}$ and $\varepsilon_{2}$. It can be seen that there is an increase in nature on heat and mass transfer rates when $R i$ increases. Therefore the mixed convection parameter plays key role in controlling temperature and concentration of the flow.

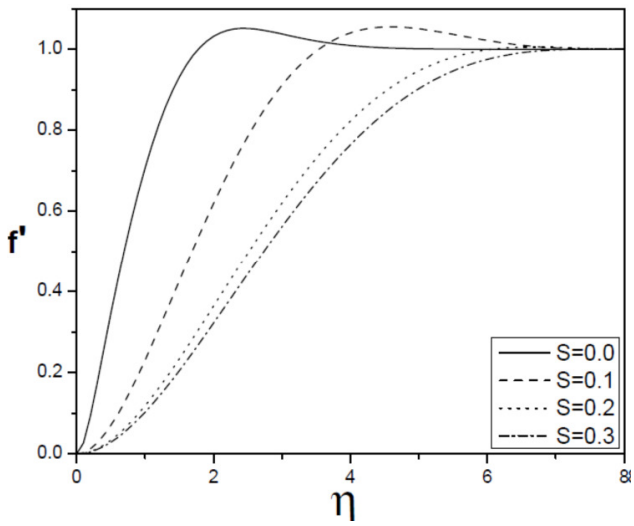

Fig. 11 Effect of couple stress parameter $S$ on velocity when $R i=2.0, \varepsilon_{1}=0.1, \varepsilon_{2}=0.1$

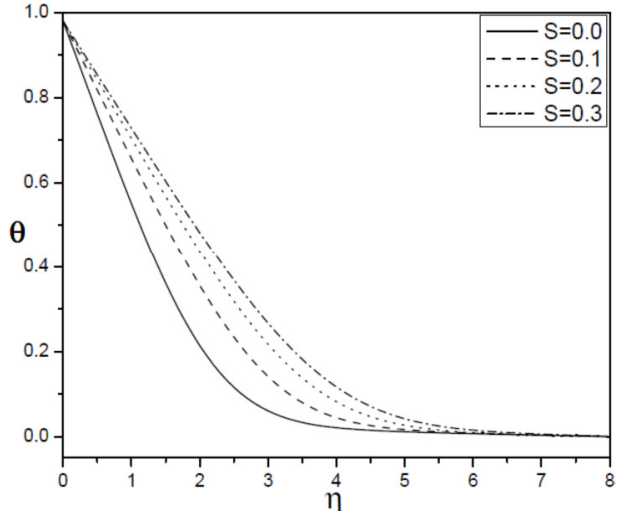

Fig. 12 Effect of couple stress parameter $S$ on Temperature at $R i=2.0, \varepsilon_{1}=0.1, \varepsilon_{2}=0.1$

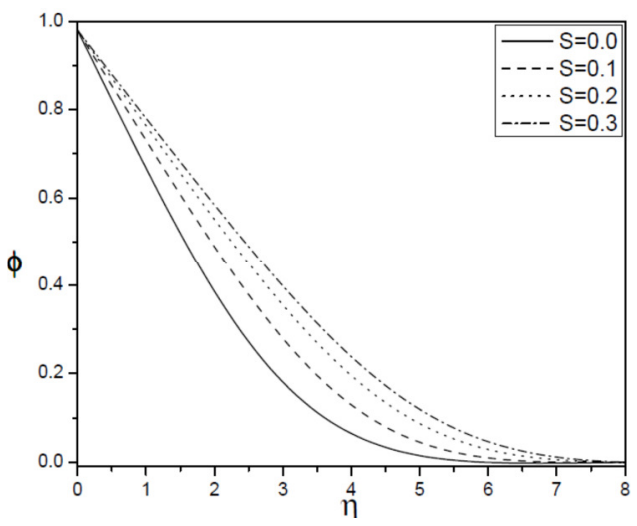

Fig. 13 Effect of couple stress parameter $S$ on concentration at $R i=2.0, \varepsilon_{1}=0.1, \varepsilon_{2}=0.1$

Table 2: Variation of non-dimensional heat mass transfer coefficients versus $\varepsilon 1$ and $\varepsilon 2$ for different values of $R i$ with $S=0.5$

\begin{tabular}{cccccccccc}
\hline \multicolumn{1}{c}{} & \multicolumn{9}{c}{$N u_{x} R e_{x}^{-1 / 2}$} \\
\hline$\varepsilon_{1}$ & $\varepsilon_{2}$ & $R i=0.5$ & $R i=1$ & $R i=1.5$ & $R i=2.0$ & $R i=0.5$ & $R i=1$ & $R i=1.5$ & $R i=2.0$ \\
\hline 0 & 0.1 & 0.21632 & 0.21972 & 0.22295 & 0.22602 & 0.18454 & 0.18684 & 0.18896 & 0.19117 \\
0.1 & 0.1 & 0.22417 & 0.22773 & 0.23111 & 0.23432 & 0.18453 & 0.18682 & 0.18896 & 0.19113 \\
0.2 & 0.1 & 0.23202 & 0.23573 & 0.23926 & 0.24261 & 0.18452 & 0.1868 & 0.18899 & 0.1911 \\
0.3 & 0.1 & 0.23987 & 0.24374 & 0.24741 & 0.2509 & 0.18451 & 0.18678 & 0.18902 & 0.19106 \\
0.4 & 0.1 & 0.24772 & 0.25173 & 0.25596 & 0.25939 & 0.1845 & 0.18676 & 0.18905 & 0.19102 \\
\hline 0.05 & 0 & 0.24389 & 0.24792 & 0.25175 & 0.25544 & 0.18386 & 0.18613 & 0.18831 & 0.19040 \\
0.05 & 0.1 & 0.24379 & 0.24773 & 0.25148 & 0.25512 & 0.18451 & 0.18677 & 0.18895 & 0.19103 \\
0.05 & 0.2 & 0.2437 & 0.24755 & 0.25121 & 0.2548 & 0.18515 & 0.18741 & 0.18958 & 0.19166 \\
0.05 & 0.3 & 0.2436 & 0.24736 & 0.25094 & 0.25449 & 0.1858 & 0.18805 & 0.19021 & 0.19228 \\
0.05 & 0.4 & 0.2435 & 0.24717 & 0.25102 & 0.25417 & 0.18644 & 0.18868 & 0.19085 & 0.19289 \\
\hline
\end{tabular}

\section{CONCLUSIONS}

In this present study, mixed convection and boundary layer analysis for couple stress fluid in presence of uniform wall temperature and concentration with thermal and solutal stratification effects is presented. The pseudo-similarity variables are used to transform the governing equations to non-dimensional form. The numerical solution is provided in terms of the profiles of dimensionless velocity, temperature, concentration and heat mass transfer rates. From this study the main findings are:
- $\quad$ Velocity, non-dimensional heat and mass transfer coefficients increases and temperature and concentration profiles are reduces as $R i$ increases.

- As $\varepsilon_{1}$ increases, the velocity, temperature distributions and nondimensional mass transfers decreases where as non-dimensional heat transfer coefficient, concentration distribution in boundary layer are increases.

- The dimensionless velocity, concentration distributions and non-dimensional heat transfer coefficients reduces and the temperature distribution, nondimensional mass transfer coefficient increases as an increase in $\varepsilon_{2}$.

- It is noticed that the presence of couple stresses in the fluid decreases the velocity and increases the temperature and concentration. 


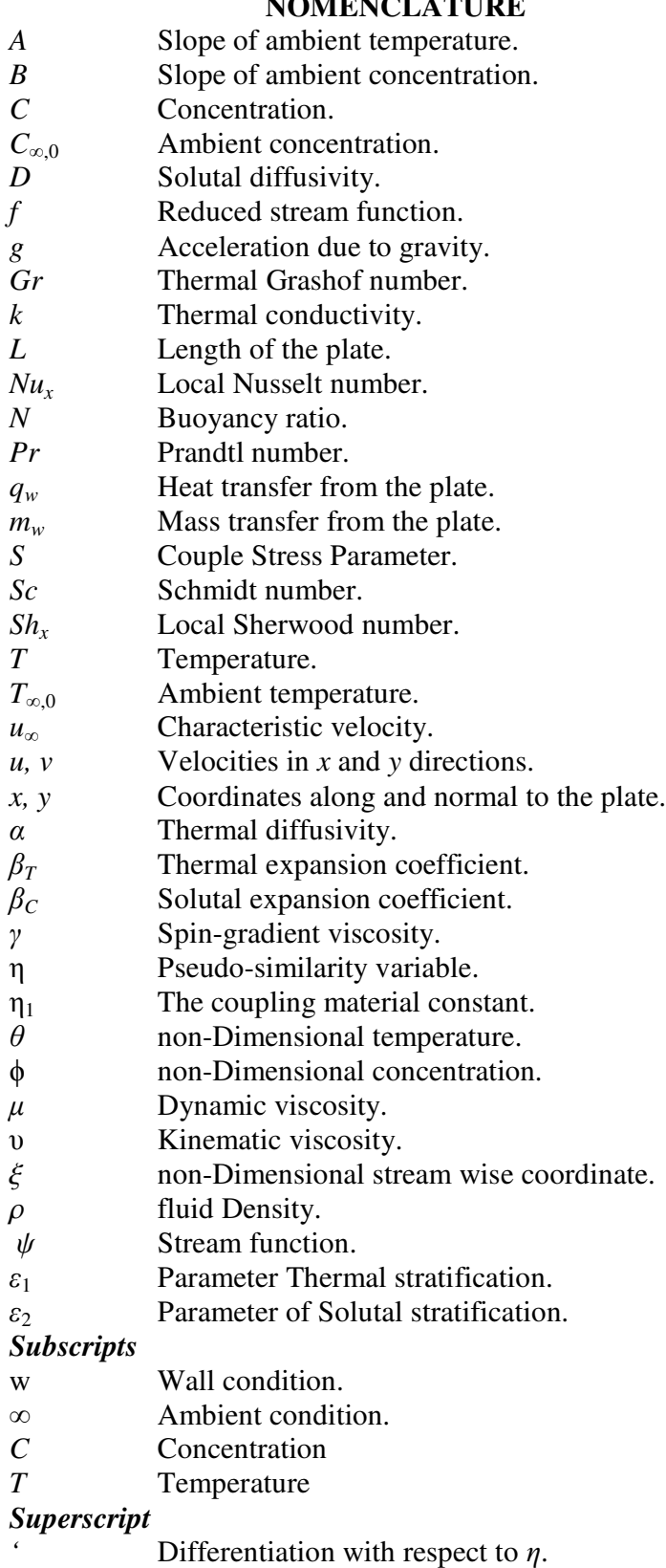

\section{REFERENCES}

Bejan, A., 1994, Convection Heat Transfer, John Wiley.

Cebeci, T. and Bradshaw, P., 1984, Physical and Computational Aspects of Convective Heat Transfer, Springer-Verlin, New York.

Hadjesfandiari, A. R., Dargush, G. F., and Hajesfandiari, A., 2013, "Consistent Skew-symmetric Couple Stress Theory for Size-Dependent Creeping Flow," Journal of Non-Newtonian Fluid Mechanics, 196, 83-94. http://DOI10.1016/j.jnnfm.2012.12.012.

Hayat, T., Mustafa, M., Iqbal, Z., and Alsaedi, A., 2013, "Stagnation-Point Flow of Couple Stress Fluid with Melting Heat Transfer," Appl. Math. Mech. -Engl. Ed, 34(2), 167-176. http://10.1007/s10483-013-1661-9.

Jaluria, Y. and Himasekhar, K., 1983, "Buoyancy Induced Two Dimensional Vertical Flows in a Thermally Stratified
Environment," Computer and Fluids, 11(1), 39-49. http://10.1016/0045-7930(83)90012-9

Lloyd, J. R. and Sparrow, E. M., 1970, "Combined Free and Forced Convective Flow on Vertical Surfaces," Int. J. Heat Mass Transfer, 13, 434-438. http://10.1016/0017-9310(70)90119-5.

Makinde, O., and Eegunjobi, A., 2013, "Entropy Generation in a Couple Stress Fluid Flow through a Vertical Channel Filled with Saturated Porous Media," Entropy, 15, 4589-4606. http://doi:10.3390/e15114589.

Murthy, P. V. S. N., Srinivasacharya, D., and Krishna, P. V. S. S. S. R., 2004, "Effect of Double Stratification on Free Convection in Darcian Porous Medium," J. Heat Transfer, 126(2), 297-300. http://doi:10.1115/1.1667525.

Muthuraj, R., Srinivas, S., and Selvi, R., 2013, "Heat and Mass Transfer Effects On MHD Flow of a Couple-Stress Fluid in a Horizontal Wavy Channel with Viscous Dissipation and Porous Medium," Heat Transfer-Asian Research, 42(5), 403-421. http://10.1002/htj.21040.

Najeeb, A. K., Mahmood, A., and Asmat, A., 2013, "Approximate Solution of Couple Stress Fluid with Expanding or Contracting Porous Channel," Engineering Computations, 30(3), $399-408$.

http://10.1108/02644401311314358.

Patil, P. and Chamkha, A. J., 2013, "Heat and Mass Transfer from Mixed Convection Flow Of Polar Fluid along A Plate In Porous Media With Chemical Reaction," Int. J. Numer. Methods Heat Fluid Flow, 23(5), 899-926. http://10.1108/HFF-03-2011-0060.

Prandtl, L., 1952, Essentials of Fluid Dynamics, London: Blackie.

Somers, E. V., 1956, "Theoretical Considerations of Combined Thermal and Mass Transfer from a Flat Plate," ASME J. Appl. Mech, 23, 295-301.

Srinivasacharya, D. and Kaladhar, K., 2012a, "Mixed Convection Flow of Couple Stress Fluid between Parallel Vertical Plates with Hall and Ion-Slip Effects," Commun Nonlinear Sci Numer Simulat, 17(6), 2447-2462. http://DOI:10.1016/j.cnsns.2011.10.006.

Srinivasacharya, D. and Kaladhar, K., 2012b, "Natural Convection Flow of A Couple Stress Fluid between Two Vertical Parallel Plates with Hall and Ion-slip Effects," Acta Mech. Sin, 28(1), 41-50.

http://10.1007/s10409-011-0523-z.

Srinivasacharya, D. and Kaladhar, K., 2013, "Analytical Solution for Hall and Ion-slip Effects on Mixed Convection Flow of Couple Stress Fluid between Parallel Disks," Math. Comput. Modell, 57(9-10), 2494-2509 http://DOI:10.1016/j.mcm.2012.12.036

Srinivasacharya, D., Pranitha, J., and RamReddy, C., 2011, "Magnetic Effect on Free Convection in a Non-Darcy Porous Medium Saturated with Doubly Stratified Power-Law Fluid," J. of the Braz. Soc. of Mech. Sci. \& Eng, 33(1), pp. 8-14. http://dx.doi.org/10.1590/S1678-58782011000100002.

Stokes, V. K., 1966, "Couple stresses in fluid," Physics of fluids, 1709-1715.

Szewczyk, A. A., 1964, "Combined Forced and FreeConvection Laminar Flow," J. Heat Transfer, C86(4), 501-507. 